\title{
Colloidal CdSe Quantum Wells with Graded Shell Composition for Low-Threshold Amplified Spontaneous Emission and Highly Efficient Electroluminescence
}

\author{
Yusuf Kelestemur, ${ }^{\dagger, \ddagger}$ Yevhen Shynkarenko, ${ }^{\dagger, \ddagger}$ Marco Anni, ${ }^{\S}{ }^{\circledR}$ Sergii Yakunin, ${ }^{\dagger, \ddagger}$ \\ Maria Luisa De Giorgi, ${ }^{\S}{ }^{\circ}$ and Maksym V. Kovalenko*, ${ }^{\dagger, \$(0)}$ \\ ${ }^{\dagger}$ Department of Chemistry and Applied Biosciences, ETH Zürich, Vladimir Prelog Weg 1, CH-8093 Zürich, Switzerland \\ ${ }^{\ddagger}$ Empa-Swiss Federal Laboratories for Materials Science and Technology, Überlandstrasse 129, CH-8600 Dübendorf, Switzerland \\ ${ }^{\S}$ Dipartimento di Matematica e Fisica "Ennio De Giorgi", Università del Salento, Via per Arnesano, 73100 Lecce, Italy
}

Supporting Information

ABSTRACT: Semiconductor nanoplatelets (NPLs) have emerged as a very promising class of colloidal nanocrystals for light-emitting devices owing to their quantum-well-like electronic and optical characteristics. However, their lower photoluminescence quantum yield (PLQY) and limited stability have hampered the realization of their outstanding luminescent properties in device applications. Here, to address these deficiencies, we present a two-step synthetic approach that enables the synthesis of core/shell NPLs with precisely controlled shell composition for engineering their excitonic properties. The proposed CdSe colloidal quantum wells possess a graded shell, which is composed of a CdS buffer layer and $\mathrm{Cd}_{x} \mathrm{Zn}_{1-x} \mathrm{~S}$ gradient layer, and exhibit bright emission (PLQY $75-89 \%)$ in the red spectral region $(634-648 \mathrm{~nm})$ with a narrow emission line width $(21 \mathrm{~nm})$. These enhanced optical properties allowed us to attain low thresholds for amplified spontaneous emission (down to $\sim 40 \mu \mathrm{J} / \mathrm{cm}^{2}$ ) under nanosecond laser excitation. We also studied the electroluminescent performance of these NPLs by fabricating solutionprocessed light-emitting diodes (LEDs). In comparison to NPL-LEDs with CdSe/CdS core/shell NPLs, which exhibit an external quantum efficiency (EQE) value of only $1.80 \%$, a significantly improved EQE value of $9.92 \%$ was obtained using graded-shell NPLs, the highest value for colloidal NPL-based-LEDs. In addition, the low efficiency roll-off characteristics of NPL-LEDs enabled a high brightness of up to $\sim 46000 \mathrm{~cd} / \mathrm{m}^{2}$ with an electroluminescence peak centered at $650 \mathrm{~nm}$. These findings demonstrate the paramount role that heterostructure engineering occupies in enhancing the optoelectronic characteristics of semiconductor NPLs toward practically relevant levels.

KEYWORDS: colloidal nanoplatelets, colloidal quantum wells, core/shell heterostructures, light-emitting devices, optical gain

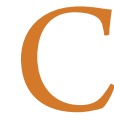

olloidal quantum wells, also known as semiconductor nanoplatelets (NPLs), have become an exciting class of semiconductor nanocrystals (NCs). ${ }^{1}$ Their quasi two-dimensional structure with atomically flat surfaces and well-defined thicknesses enables access to distinctly different optical properties. ${ }^{2,3}$ They exhibit narrower emission line widths, larger absorption cross sections, giant oscillator strength, and suppressed Auger recombination as compared to spherically shaped NCs. ${ }^{3-6}$ These exciting properties have already led to the demonstration of low-threshold amplified spontaneous emission (ASE) with high gain coefficients using CdSe core-only NPLs ${ }^{7-9}$ and to the fabrication of lightemitting diodes (LEDs) with high color purity through the use of CdSe/CdS core/shell and core/crown NPLs as emissive layers. ${ }^{10,11}$ Nevertheless, the realization of the outstanding performance of these NPLs in optoelectronic applications has been hampered by their low photoluminescence quantum yield (PLQY) and limited photostability.

To enhance the PLQY and the photostability of the NPLs, two different heterostructures have been widely investigated: core/crown NPLs ${ }^{12}$ and core/shell NPLs. ${ }^{13}$ In the former case, the peripheries of the core NPLs are covered by the laterally extended crown layer without the deposition of shell material onto the large surfaces of the NPLs. Currently, core/crown NPLs with different compositions such as $\mathrm{CdSe} / \mathrm{CdS},{ }^{12} \mathrm{CdSe} /$

Received: July 5, 2019

Accepted: November 26, 2019

Published: November 26, 2019 
$\mathrm{CdTe}^{14} \mathrm{CdSe} / \mathrm{CdSe}_{x} \mathrm{Te}_{1-x}{ }^{15}$ and $\mathrm{CdS} / \mathrm{CdSe}^{16}$ have been successfully synthesized and studied, showing improved PLQY. Nevertheless, the unpassivated larger surfaces determine the optoelectronic characteristics of core/crown NPLs. In the case of core/shell NPLs, the growth of a shell layer in both the lateral and vertical directions results in complete passivation for the core NPLs. The first examples of core/shell NPLs were synthesized through colloidal atomic layer deposition (cALD), ${ }^{17}$ whereby Cd and S layers were successively deposited in a multistep process assisted by ligand-exchange reactions and phase-transfer procedures. However, the c-ALD approach is performed at room temperature, and this results in the decreased crystallinity, lower PLQY, and limited photostability for the synthesized core/shell NPLs, including both CdSe/ $\mathrm{CdS}^{18}$ and $\mathrm{CdSe} / \mathrm{ZnS}{ }^{19}$ Although passivating the inner CdSe core NPLs with a CdS crown layer prior to the growth of a $\mathrm{CdS}^{20}$ and $\mathrm{Cd}_{x} \mathrm{Zn}_{1-x} \mathrm{~S}^{21}$ shell have been reported to enhance the PLQY, the resulting core/crown/shell NPLs have still suffered from decreased crystallinity and stability suggesting that a different synthetic approach for shell growth is necessary.

Recently, high-temperature approaches to shell growth have been reported for the synthesis of core/shell NPLs (with $\mathrm{CdS}^{22}$ or $\mathrm{ZnS}^{23}$ shells) to overcome the limitations of lowtemperature c-ALD. In these studies, a $\mathrm{Cd}$ or $\mathrm{Zn}$ precursor was added already at the beginning of the synthesis to preserve the morphology of the CdSe NPLs. If these precursors were added later in the course of heating the CdSe NPLs in the presence of commonly used reaction mixtures, such as octadecene (ODE) and oleylamine, detrimental effects of dissolution and etching of NPLs took place. ${ }^{22,24}$ However, the addition of precursors at the beginning of the synthesis makes it challenging to control and tune the composition of the shell layer, which is necessary for properly engineering the optical properties of NPLs. For example, CdSe/CdS core/shell NPLs synthesized in this manner possessed a narrow emission line width (down to $\sim 18 \mathrm{~nm}$ ) but with a moderate PLQY of $50-60 \% .^{22}$ This moderate PLQY may be attributed to the improper confinement of the electron with the CdS shell, which enables the interaction of the electron with surface trap sites. In the case of $\mathrm{CdSe} / \mathrm{ZnS}$ core/shell NPLs, the growth of a thin layer of $\mathrm{ZnS}$ as the shell yields much better confinement for both excited charge carriers, and the PLQY of core/shell NPLs was observed to be significantly improved $(>90 \%){ }^{23}$ However, because of the high lattice mismatch, or the difference in unit cell constants, between CdSe and $\mathrm{ZnS}$ crystals, the growth of thick $\mathrm{ZnS}$ shells results in a nonuniform shape, broadened photoluminescence (PL) emission line widths, and decreased PLQYs. ${ }^{23,25}$ As a result, core/shell NPLs with specifically designed shell compositions such as alloys and/or gradient $\mathrm{Cd}_{x} \mathrm{Zn}_{1-x} \mathrm{~S}$ shells remain important targets. In the course of publishing this work, Rossinelli et al. and Altintas et al. have reported core/shell NPLs having graded and alloyed shell compositions, albeit with a different synthesis procedures. ${ }^{26,27}$

Here, we propose a two-step synthetic approach that enables the synthesis of CdSe-based core/shell NPLs with compositionally adjustable $\mathrm{Cd}_{x} \mathrm{Zn}_{1-x} \mathrm{~S}$ shells. With the continuous injection of shell precursors at high reaction temperatures, we succeeded in growing highly crystalline $\mathrm{CdS}$ and $\mathrm{Cd}_{x} \mathrm{Zn}_{1-x} \mathrm{~S}$ shell layers. The combined effect of the CdS buffer and $\mathrm{Cd}_{x} \mathrm{Zn}_{1-x} \mathrm{~S}$ gradient shell allows for an improved PLQY (up to $89 \%$ ), as compared to CdSe/CdS core/shell NPLs, and a narrow emission line width of $21 \mathrm{~nm}$. Additionally, we studied the optical gain characteristics of these NPLs under nano- second laser excitation and attained some of the lowest ASE thresholds (down to $\sim 40 \mu \mathrm{J} / \mathrm{cm}^{2}$ ) with high photostability. We then fabricated LEDs using core/shell NPLs as an emitter. With our optimized device architecture (ITO/PEDOT:PSS/ $\mathrm{PVK} / \mathrm{NPLs} / \mathrm{ZnO} / \mathrm{Ag}$ ), we achieved an external quantum efficiency (EQE) value of $9.92 \%$ with a brightness of up to $46000 \mathrm{~cd} / \mathrm{m}^{2}$; such an EQE is a record among colloidal NPLbased LEDs. Our findings indicate that the heterostructure engineering of colloidal quantum wells has a profound impact on the realization of their exceptional excitonic features: enabling superior performance in optoelectronic applications.

\section{RESULTS/DISCUSSION}

In this study, we present a high-temperature approach toward shell growth to obtain highly efficient core/shell NPLs. Instead of adding the precursors at the beginning of the synthesis, ${ }^{22,23}$ the shell growth was performed by continuously injecting precursors, enabling precise control over the final shell composition (Scheme 1). To mitigate problems pertaining to

Scheme 1. Synthesis of CdSe-Based Colloidal Quantum Wells with Adjustable Shell Composition at High Reaction Temperature by Continuous Injection of Precursors ${ }^{a}$

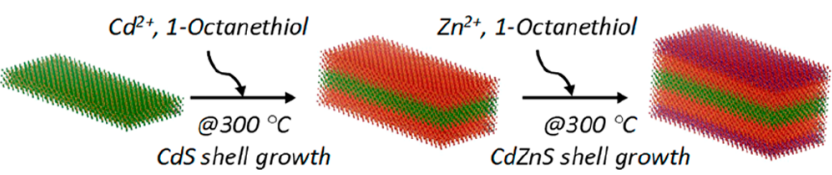

${ }^{a}$ While continuous injection of $\mathrm{Cd}$ and $\mathrm{S}$ precursors enables the growth of $\mathrm{CdS}$ shell, the replacement of $\mathrm{Cd}$ precursor with $\mathrm{Zn}$ at later stage of shell growth allows us to obtain core/shell NPLs with a graded shell.

the dissolution and etching of core NPLs prior to shell growth, we thought of shortening the annealing time of the CdSe NPLs and limiting it only to shell growth. We thus adopted a method wherein the shell precursors are first added at a high temperature to the pure solvent. The concentrations of these precursors are still too low to induce any substantial nucleation of CdS NCs, since homogeneous nucleation requires higher supersaturation than that of heterogeneous nucleation. We then inject the CdSe seed NPLs into such a solution to initiate the growth of the $\mathrm{CdS}$ shell. Further addition of $\mathrm{Cd}$ and $\mathrm{S}$ precursors maintains the supersaturation needed for shell growth.

As core NPLs, four-monolayer thick (4 ML) CdSe NPLs (composed of five layers of $\mathrm{Cd}$ atoms and four layers of Se atoms with an emission centered at $513 \mathrm{~nm}$ ) were prepared according to published protocol. ${ }^{12}$ To grow a shell, we used $\mathrm{ODE}$ as the solvent and heated the reaction mixture to $300{ }^{\circ} \mathrm{C}$ at a rate of $10-15{ }^{\circ} \mathrm{C} / \mathrm{min}$ under a nitrogen atmosphere. When the temperature reached $150{ }^{\circ} \mathrm{C}$, we began to inject shell precursors at a constant rate of $7 \mathrm{~mL} / \mathrm{h}$. After the injection of $0.2 \mathrm{~mL}$ of shell precursors, CdSe core NPLs dissolved in ODE were swiftly added to the reaction mixture; the shell growth proceeded at $300{ }^{\circ} \mathrm{C}$ (see the Experimental Section for a detailed description). During the optimization of the shell growth, we also tested the addition of $\mathrm{CdSe}$ core NPLs to the reaction solution at the beginning of the synthesis. For instance, initial addition of CdSe core NPLs to the pure solvent resulted in the aggregation and destabilization of NPLs during heating. An attempt to mitigate this issue by introducing additional oleic acid or oleylamine as ligands to 


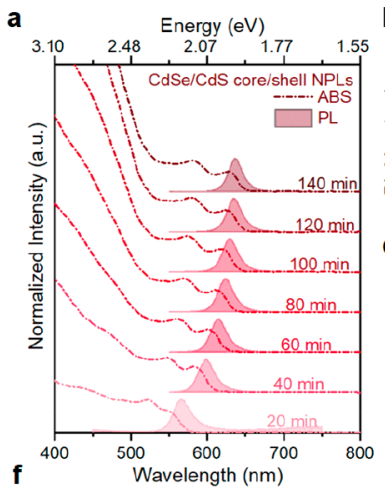

b
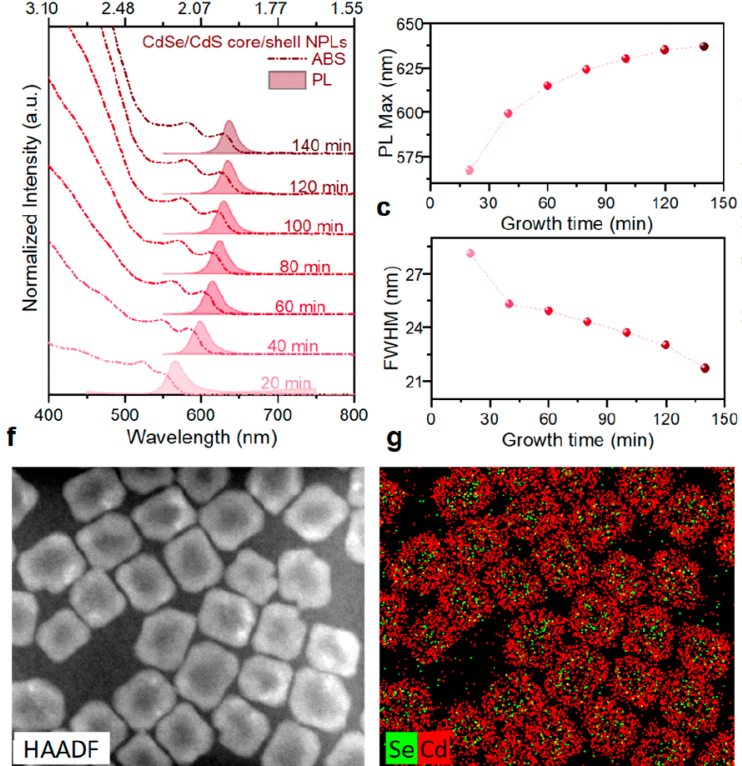
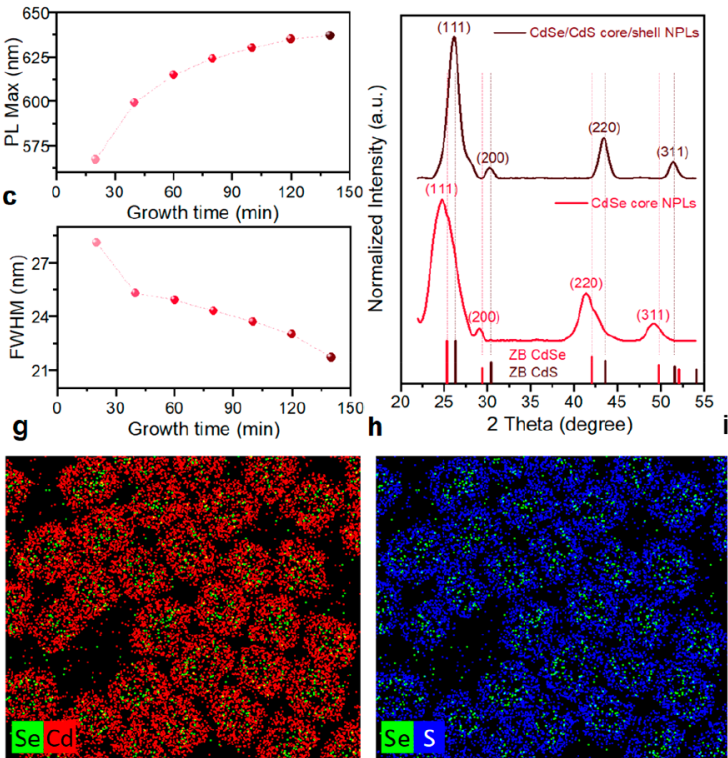

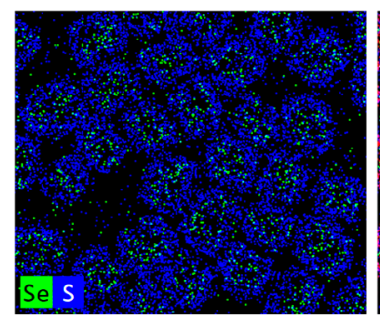

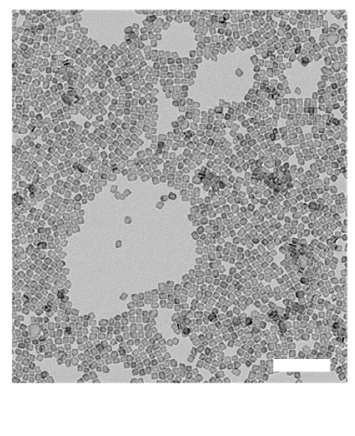

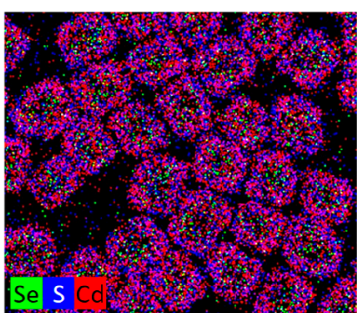

Figure 1. Optical and structural characterization of CdSe/CdS core/shell NPLs. (a) Normalized absorbance and PL spectra of core/shell NPLs taken at different stages of shell growth. The changes in the PL peak position (b) and fwhm value (c) of corresponding core/shell NPLs. (d) X-ray diffraction patterns of CdSe core only and CdSe/CdS core/shell NPLs with the reference lines showing the peaks of the bulk zinc blende CdSe (red) and CdS (brown). (e) TEM image of core/shell NPLs with a scale bar of $200 \mathrm{~nm}$. (f) HAADF-STEM image of core/shell NPLs and their high-resolution elemental mapping $(g-i)$.

the reaction mixture deteriorated the resulting properties of core/shell NPLs (nonuniform shapes and lower PLQY). As a remedy to these issues, we decided to add the CdSe core NPLs at a later stage of the shell growth into the $\mathrm{Cd}$ - and S-rich reaction mixture. The apparent improvement is likely due to the presence of Cd-oleate as the sole ligand in the reaction mixture, which also acts as a Z-type ligand. ${ }^{28}$ This provides improved colloidal stability for the CdSe NPLs and lessens the dissolution and etching rate.

First, we studied the synthesis of $\mathrm{CdSe} / \mathrm{CdS}$ core/shell NPLs using Cd-oleate and octanethiol as the cadmium and sulfur precursors, respectively. During the initial stages of shell growth, we observed significantly broadened absorption and PL peaks, suggesting the incomplete and nonuniform growth of the CdS shell (Figure $1 \mathrm{a}-\mathrm{c}$ ). The continuous injection of shell precursors resulted in strongly red-shifted emission (up to $\sim 640 \mathrm{~nm}$ ) - due to the reduced quantum confinement that is concomitant with increased CdS shell thickness-and a reduced full-width-half-maximum (fwhm) of emission (down to $21 \mathrm{~nm}$ ), indicating the improved uniformity of CdS shell. However, the emission line width of $\mathrm{CdSe} / \mathrm{CdS}$ core/shell NPLs $(\sim 66 \mathrm{meV})$ is notably broader than the starting CdSe core NPLs $(\sim 39 \mathrm{meV})$. This behavior has already been observed for $\mathrm{CdSe} / \mathrm{CdS}$ core/shell NPLs synthesized with different approaches, and it is attributed to the enhanced exciton-phonon coupling that occurs with the formation of the CdS shell. ${ }^{29}$

After the synthesis, the resulting CdSe/CdS core/shell NPLs can be easily separated from the side products by centrifugation. From the X-ray powder diffraction patterns presented in Figure 1d, it can be observed that the diffraction peaks of the core/shell NPLs are shifted toward higher angles and that they become narrower as compared to those of the core CdSe NPLs, indicating the formation of a CdS shell with improved crystallinity. While the main diffraction peaks of the core/shell NPLs were assigned to the initial zinc-blende crystal structure, the shoulder of the (111) peak indicates an additional reflection from the wurtzite phase. This demonstrates the wurtzite/zinc-blende polytypic characteristics of the resulting core/shell NPLs. Nonetheless, the synthesized core/ shell NPLs exhibit a uniform size distribution with sharp edge boundaries (Figure 1e). The lateral dimensions of the CdSe/ CdS core/shell NPLs were found to increase from $19.0 \pm 2.9$ by $10.2 \pm 1.0 \mathrm{~nm}$ to $23.5 \pm 2.5$ by $19.2 \pm 1.8 \mathrm{~nm}$ after shelling. This indicates a lack of dissolution and etching related to NPL edge erosion. This is in contrast to a previous report, where Rossinelli et al. observed a decrease in the lateral size of core/ shell NPLs due to dissolution and etching. ${ }^{22}$ From the highresolution elemental mapping of the synthesized NPLs (Figure $1 \mathrm{f}-\mathrm{i}$ ), we obtained further evidence of the formation of a core/ shell heterostructure, where CdS shell layers were grown in both lateral and vertical directions, enclosing the inner CdSe core.

This approach reproducibly yields $\mathrm{CdSe} / \mathrm{CdS}$ core/shell NPLs with emission peak wavelengths within the range of 632-646 nm and narrow emission line widths down to $21 \mathrm{~nm}$ (Table S1). However, these core/shell NPLs exhibit a moderate PLQY (45-60\%), which is similar to the previous report on $\mathrm{CdSe} / \mathrm{CdS}$ core/shell NPLs synthesized at a high reaction temperature. ${ }^{22}$ Although very high PLQYs (>80\%) have been routinely obtained with spherical CdSe/CdS core/ shell NCs, ${ }^{30,31}$ the moderate PLQY of these core/shell NPLs may be attributed to the improper confinement of electrons by the CdS shell, the increased surface-to-volume ratio of NPLs, and the nonuniform growth of the $\mathrm{CdS}$ shell. The varying thickness of the CdS shell can be seen from the high-angle annual dark-field scanning transmission electron microscopy (HAADF-STEM) image that shows the contrast difference between the center and the edges of the NPLs. While thicker CdS shells were observed on the edges of NPLs, the thickness 

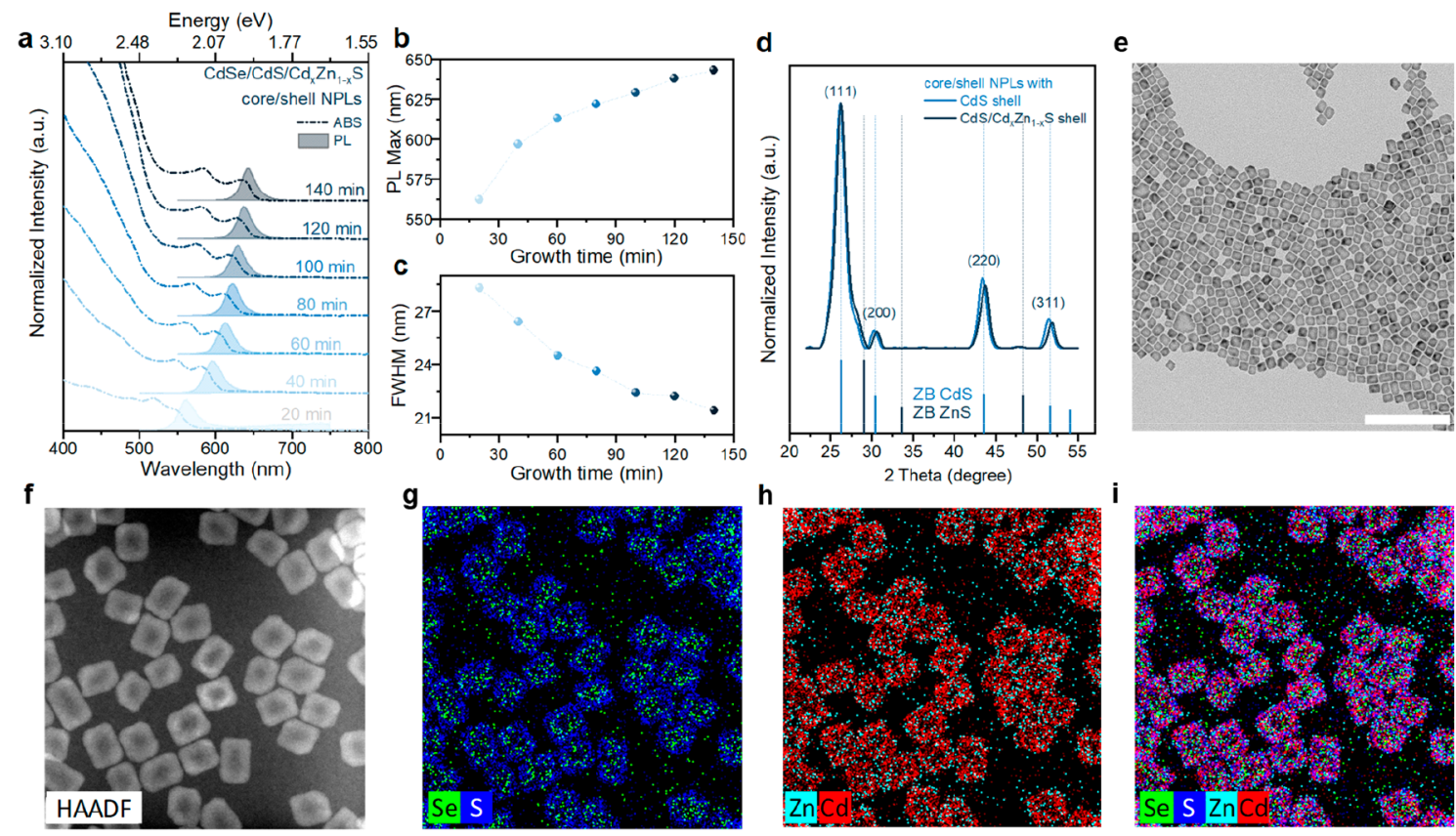

Figure 2. Optical and structural characterization of $\mathrm{CdSe} / \mathrm{CdS} / \mathrm{Cd}_{x} \mathrm{Zn}_{1-x} \mathrm{~S}$ core/shell NPLs. (a) Normalized absorbance and PL spectra of core/shell NPLs taken at different time intervals during the synthesis. The changes in the PL peak position (b) and fwhm value (c) of corresponding core/shell NPLs with respect to shell growth time. (d) X-ray diffraction patterns of core/shell NPLs with CdS and CdS/ $\mathrm{Cd}_{x} \mathrm{Zn}_{1-x} \mathrm{~S}$ shell together with the dashed lines showing the reference peaks belonging to the bulk zinc blende CdS (blue) and $\mathrm{ZnS}$ (dark blue). (e) TEM image of core/shell NPLs with a scale bar of $200 \mathrm{~nm}$. (f) HAADF-STEM image of core/shell NPLs and their high-resolution elemental mapping $(g-i)$.

of CdS shell layers decreased toward the center of the core NPLs: this may deteriorate their performance. Furthermore, electrons within the $\mathrm{CdSe} / \mathrm{CdS}$ core/shell NPLs face a lower energy barrier, which allows electrons to delocalize over the whole structure, and this leads to enhanced interactions with surface trap sites and decreases their PLQY. Therefore, we designed a shell structure composed of a CdS buffer interlayer, which serves to reduce the crystal strain with the subsequent $\mathrm{Cd}_{x} \mathrm{Zn}_{1-x} \mathrm{~S}$. This graded shell then provides better confinement of the carriers.

Such a CdS $/ \mathrm{Cd}_{x} \mathrm{Zn}_{1-x} \mathrm{~S}$ shell was obtained from conditions derived from those used to prepare a pure CdS shell. After the injection of a certain amount of cadmium and sulfur precursors, which yields a buffer CdS interlayer, the cadmium precursor is replaced with the zinc precursor, and the injection continues. At this point, both $\mathrm{Cd}$ and $\mathrm{Zn}$ precursors coexist in solution, yielding a $\mathrm{Cd}_{x} \mathrm{Zn}_{1-x} \mathrm{~S}$ shell (see the Experimental Section). The absorption and PL spectra from aliquots taken at different reaction times during the synthesis of core/shell NPLs are presented in Figure 2a. They also exhibit strongly red-shifted excitonic features similar to the $\mathrm{CdSe} / \mathrm{CdS}$ core/ shell NPLs. However, the formation of the $\mathrm{Cd}_{x} \mathrm{Zn}_{1-x} \mathrm{~S}$ shell provides stronger confinement for excited charge carriers and decreases their interaction with surface trap sites leading to a higher solution PLQY of up to $89 \%$. Furthermore, the $\mathrm{Cd}_{x} \mathrm{Zn}_{1-x} \mathrm{~S}$ shell preserved the narrower emission line widths $(21 \mathrm{~nm})$, which may be attributed to a reduction in strain.

The growth of the additional $\mathrm{Cd}_{x} \mathrm{Zn}_{1-x} \mathrm{~S}$ shell was confirmed by X-ray powder diffraction and energy dispersive X-ray spectroscopy (EDS) measurements. The diffraction peaks from the core/shell NPLs (Figure $2 \mathrm{~d}$ ), which possess an additional $\mathrm{Cd}_{x} \mathrm{Zn}_{1-x} \mathrm{~S}$ shell, are shifted to higher angles with respect to those that have a pure CdS shell. High-resolution elemental mapping of the resulting core/shell NPLs also points to the formation of a core/shell heterostructure with a $\mathrm{Cd}_{x} \mathrm{Zn}_{1-x} \mathrm{~S}$ shell. EDS measurements yield a $\mathrm{Zn} / \mathrm{Cd}$ ratio of $\sim 0.13$ in the shell, which is lower than the injected shell precursor ratio (0.4; Figure S2). Similar behavior was observed in the synthesis of core/shell NCs with a $\mathrm{Cd}_{x} \mathrm{Zn}_{1-x} \mathrm{~S}$ shell, showing the reduced reactivity of $\mathrm{Zn}$-oleate as compared to $\mathrm{Cd}$ oleate. $^{32,33}$ We also synthesized and analyzed core/shell NPLs that have shells consisting of either alloyed $\mathrm{Cd}_{x} \mathrm{Zn}_{1-x} \mathrm{~S}$ or pure $\mathrm{ZnS}$ by adjusting the precursor ratio of the injection (Figure S3). The core/shell NPLs with graded shells exhibited the highest PLQY among the synthesized NPLs having various other shell layers. This further proves the importance of heterostructure engineering as a way to realize improved optoelectronic properties.

We then investigated the optical gain performance of these core/shell NPLs. Colloidal NPLs have gained prominence in recent literature as optical gain media for lasing applications. $^{7,8,34}$ In comparison to spherical NCs, NPLs have two important advantages: their larger physical volume can significantly improve absorption cross sections, and their quasi-two-dimensional electronic structure leads to the suppression of Auger recombination. ${ }^{34}$ Given these appealing properties, the optical gain and lasing performance of colloidal NPLs has been extensively studied. ${ }^{35-38}$ For example, very low optical gain thresholds $\left(\sim 6 \mu \mathrm{J} / \mathrm{cm}^{2}\right.$, at $\left.635 \mathrm{~nm}\right)$ have been obtained with $\mathrm{CdSe} / \mathrm{CdS}$ core/shell NPLs. ${ }^{7}$ We initially studied the optical gain performances of our core/shell NPLs under femtosecond laser excitation at $400 \mathrm{~nm}$ and obtained low ASE thresholds of $\sim 3.0 \mu \mathrm{J} / \mathrm{cm}^{2}$ for both graded and CdSonly shells (Figure S4). These results are commensurate with the value of $2.4 \mu \mathrm{J} / \mathrm{cm}^{2}$ for $\mathrm{CdSe} / \mathrm{CdZnS}$ core/shell NPLs 
synthesized at high reaction temperatures, as just reported by Altintas et al. ${ }^{27}$

We then examined the optical gain characteristics under nanosecond pulses. The excitation intensity dependent PL spectra of core/shell NPLs having a pure CdS and an additional $\mathrm{Cd}_{x} \mathrm{Zn}_{1-x} \mathrm{~S}$ shell under nanosecond laser excitation are illustrated in Figure 3a,b. In the case of $\mathrm{CdSe} / \mathrm{CdS}$ core/
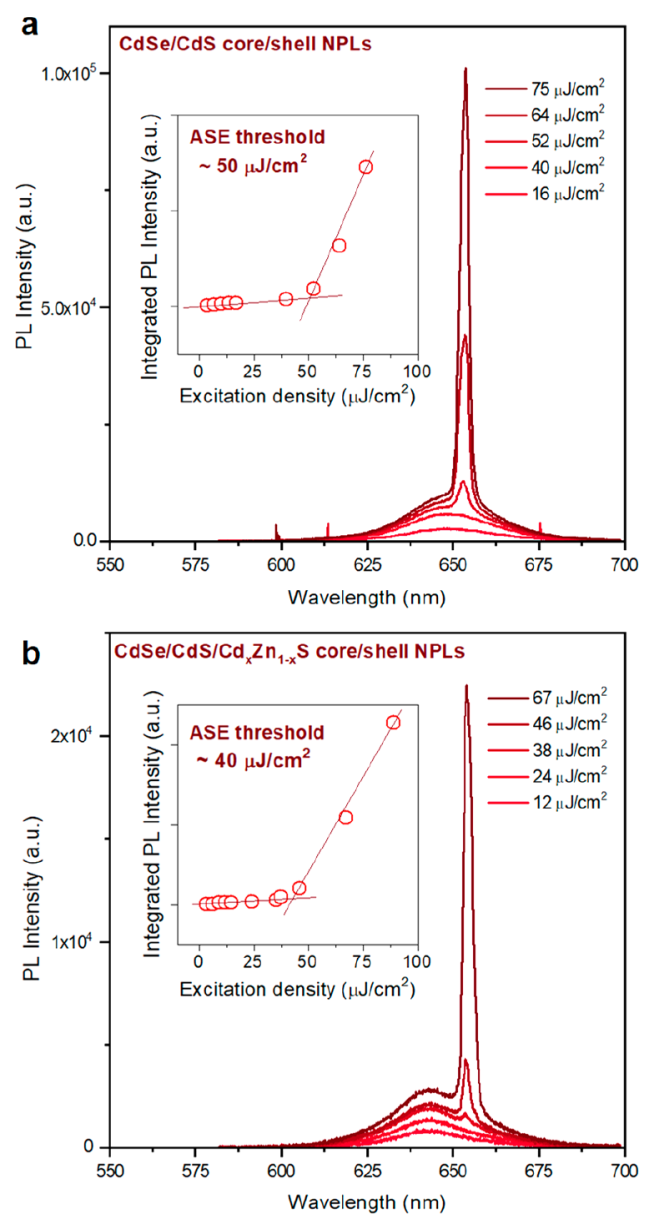

Figure 3. Optical gain performances of core/shell NPLs. PL spectra of CdSe/CdS (a) and CdSe/CdS $/ \mathrm{Cd}_{x} \mathrm{Zn}_{1-x} \mathrm{~S}(\mathrm{~b})$ core/shell NPLs at different excitation densities together with the inset showing the integrated PL intensity as a function of excitation density.

shell NPLs, an ASE band centered at $653 \mathrm{~nm}$ emerges when the excitation intensity exceeded $\sim 50 \mu \mathrm{J} / \mathrm{cm}^{2}$, which is slightly red-shifted with respect to the spontaneous emission peak $(648 \mathrm{~nm})$. As the excitation intensity increases further, the ASE peak becomes more intense, and its emission line width narrows $(\sim 2.5 \mathrm{~nm})$. The red-shifted ASE peak is attributed to a biexcitonic gain mechanism, which suggests an attractive exciton-exciton (X-X) interaction in the $\mathrm{CdSe} / \mathrm{CdS}$ core/shell NPLs owing to their Type-I-like band alignment. ${ }^{39,40}$ In the case of core/shell NPLs with a graded shell, the ASE threshold is reduced to $\sim 40 \mu \mathrm{J} / \mathrm{cm}^{2}$, and the ASE peak is centered at 654 $\mathrm{nm}$ and, hence, is also red-shifted with respect to the spontaneous emission peak $(\sim 642 \mathrm{~nm})$. This additional shift in the ASE peak also supports the growth of a $\mathrm{Cd}_{x} \mathrm{Zn}_{1-x} \mathrm{~S}$ shell layer, since the formation of shell layer with a larger bandgap provides better confinement of electrons and holes (compared to a pure CdS shell) and increases the overlap of their wave functions. Therefore, the resulting core/shell NPLs exhibit stronger Type-I character with enhanced, attractive X-X interactions, resulting in the greater redshift of the ASE peak. We also measured the ASE stability from core/shell NPLs at an excitation density that was twofold higher than their ASE thresholds under ambient conditions and in vacuum (see results and further discussion in Figure S5).

The reduced ASE thresholds of core/shell NPLs can be attributed to the increased physical volume of NPLs, since this leads to an enhanced absorption cross-section and suppressed Auger recombination rates as compared to pristine CdSe core NPLs. Furthermore, a very high ASE threshold was observed for CdSe core NPLs $\left(\sim 1.8 \mathrm{~mJ} / \mathrm{cm}^{2}\right)$ under similar excitation conditions, and this emphasizes the decisive effect of the shell on reducing ASE thresholds.

We then investigated the electroluminescent characteristics of the core/shell NPLs by fabricating solution-processable LEDs. Since the first demonstration of colloidal NC-LEDs, ${ }^{41}$ enormous efforts have been devoted to the development of highly efficient, bright, and stable electroluminescent devices by using free-standing inorganic NCs as a promising alternative to organic emitters. ${ }^{42,43}$ In recent studies, colloidal NC-LEDs with very high EQE values reaching $\sim 20 \%{ }^{44}$ and brightness exceeding $10000 \mathrm{~cd} / \mathrm{m}^{2}$ in the blue, ${ }^{45}$ green, $^{46}$ and $\mathrm{red}^{47}$ spectral regions have been reported. Despite this great progress, colloidal NC-LEDs have faced several challenges including efficiency roll-off, broad electroluminescence, and limited light out-coupling efficiency. ${ }^{48}$ Generally, colloidal NCbased LEDs exhibit their peak EQE values at low current densities $\left(\sim 1-10 \mathrm{~mA} / \mathrm{cm}^{2}\right) .{ }^{47}$ Increasing the current density in NC-based LEDs usually results in a significant drop in EQE-this is known as efficiency roll-off, and this degrades device performance by enhancing nonradiative Auger recombination and device heating. In addition, the size distribution of NCs within an ensemble or a solution causes further broadening of the emission line width due to inhomogeneous broadening. This broadening limits the achievable color gamut for high-color-quality displays. ${ }^{42}$ Moreover, the isotropic emission of spherically shaped colloidal NCs restricts the maximum attainable EQE value of NC-LEDs to $\sim 20 \%$ due to the limited light-outcoupling efficiency. At this point, anisotropically shaped colloidal NPLs with suppressed Auger recombination, narrow emission line width, and aligned emission dipoles have arisen as very promising candidates for developing high-performance LEDs. ${ }^{49,50}$

The device architecture of the fabricated LEDs is depicted in Figure 4a, where poly(9-vinylcarbazole) (PVK) and $\mathrm{ZnO}$ nanoparticles (NPs) were employed as hole and electron injection layers, respectively. It is known that the direct contact of NCs to $\mathrm{ZnO}$ can quench emission; ${ }^{44}$ thus, we analyzed the in-film emission characteristics of core/shell NPLs spin-coated on bare and $\mathrm{ZnO}$-coated quartz substrates. We found that the solution PLQY of CdSe/CdS core/shell NPLs (48\%) dropped to $40 \%$ and $22 \%$ on bare and $\mathrm{ZnO}$-coated quartz substrates, respectively (Figure S6). This significant drop in PLQY is attributed to the nonradiative energy transfer between NPLs and exciton quenching at the interface between the NPLs and the $\mathrm{ZnO}$ NPs. This conclusion is supported by time-resolved PL measurements showing accelerated decay kinetics for core/ shell NPLs on $\mathrm{ZnO}$ (Figure S7). We observed a similar trend in the PLQY for core/shell NPLs with a graded shell. However, they exhibit a substantially higher PLQY of 53\% on 
a

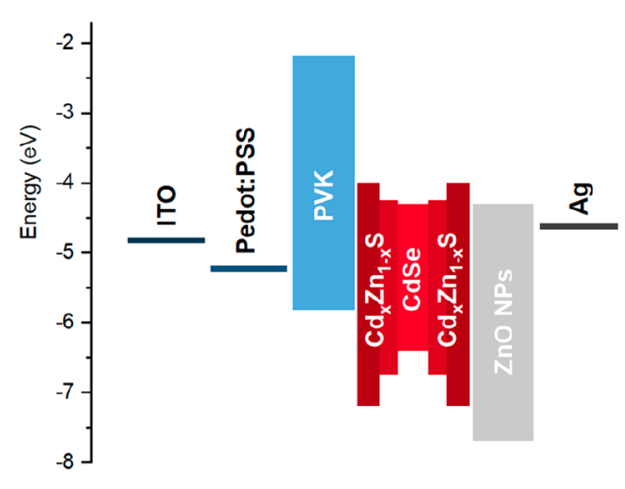

c

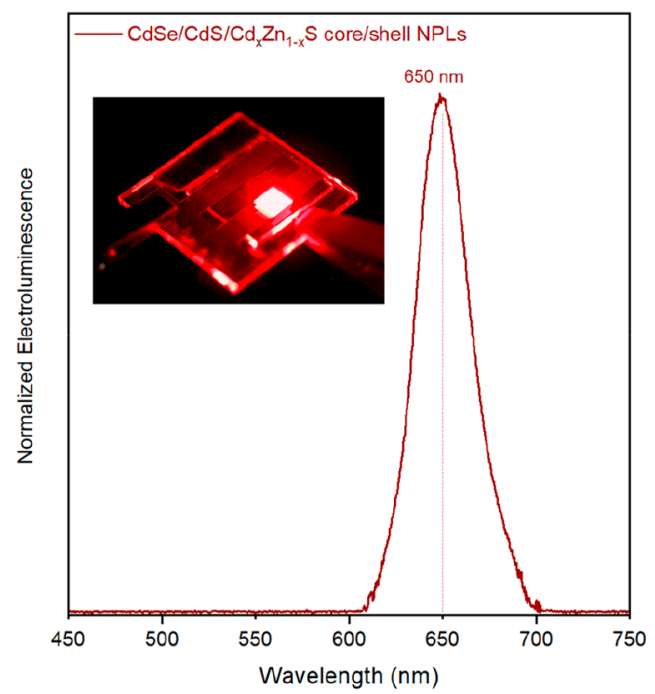

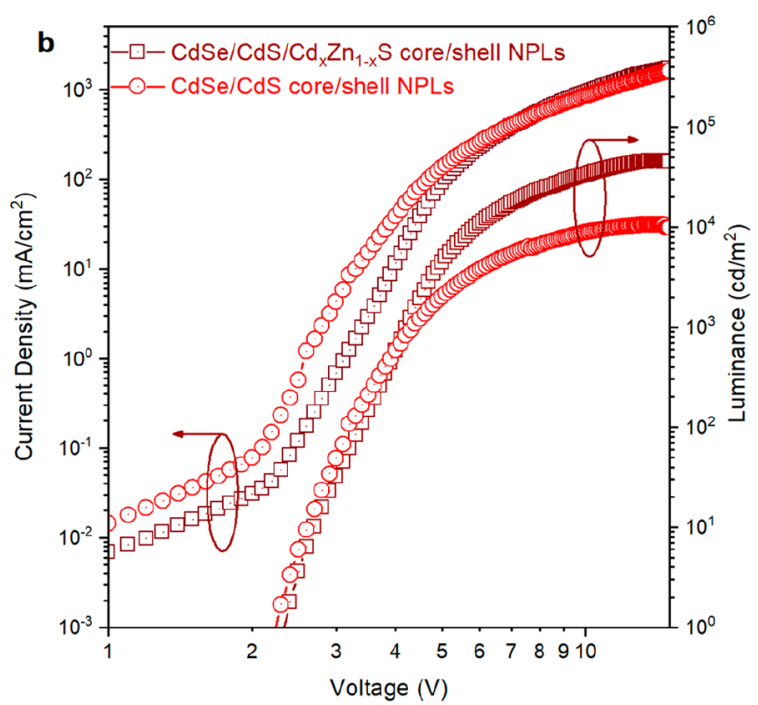

d

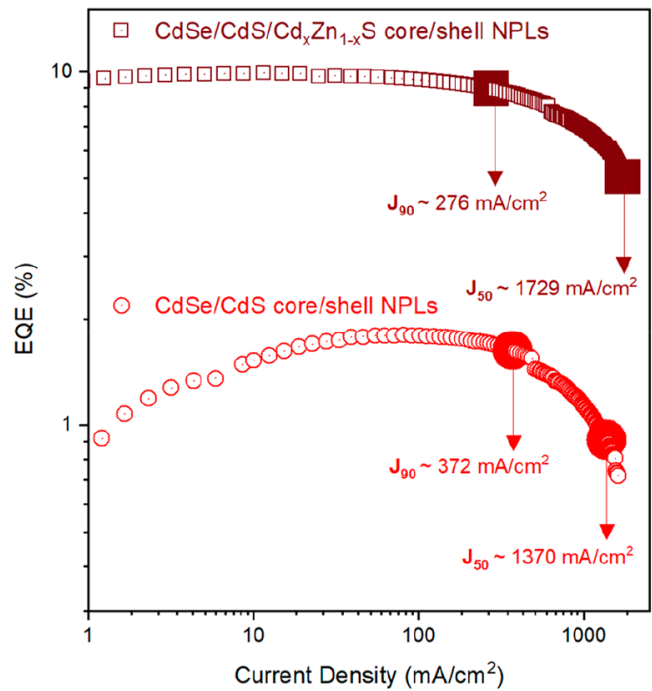

Figure 4. LED performances of core/shell NPLs. (a) Energy band diagram of the optimized device structure. (b) The current densityluminance-voltage characteristics of the LEDs employing core/shell NPLs with CdS and $\mathrm{CdS} / \mathrm{Cd}_{x} \mathrm{Zn}_{1-x} \mathrm{~S}$ shell layers as emitters. (c) Electroluminescence spectra of NPL-LEDs using CdSe/CdS/Cd $\mathrm{Zn}_{1-x} \mathrm{~S}$ core/shell NPLs as an emitter with the inset showing a photo of the fabricated LED in operation. (d) EQE vs current density representation of NPL-LEDs, with $J_{90}$ and $J_{50}$ current density values corresponding to a $10 \%$ and $50 \%$ drop in the peak $\mathrm{EQE}$ at high current densities, respectively.

$\mathrm{ZnO}$ NPs, suggesting the suppressed exciton quenching with the growth of the additional confinement layer. From PLQY measurements, the maximum attainable EQE values of the devices are estimated to be $10.6 \%$ for NPLs with graded shells and $4.4 \%$ for those with pure CdS shells, assuming near-unity charge injection and an out-coupling efficiency of $20 \%$.

The current density-luminance-voltage characteristics of the best-performing devices with both kinds of shells are presented in Figure 4b. The devices exhibit a steep increase in current density and luminance behavior after the turn-on voltage of $\sim 2.3 \mathrm{~V}$, showing behavior typical for diodes in forward bias. For NPL-based LEDs fabricated with CdSe/CdS core/shell NPLs, we observed a peak EQE of $1.80 \%$ at a current density of $80.6 \mathrm{~mA} / \mathrm{cm}^{2}$ with a maximum brightness of $10650 \mathrm{~cd} / \mathrm{m}^{2}$. The low EQE values of LEDs with NPLs that have a pure $\mathrm{CdS}$ shell can be attributed to the similar conduction band edges of $\mathrm{CdS}$ and $\mathrm{ZnO}$, causing imbalanced charge injection. With the addition of a $\mathrm{Cd}_{x} \mathrm{Zn}_{1-x} \mathrm{~S}$ shell layer, we introduced an additional barrier to the injection of electrons and provided proper surface passivation. By doing so, we greatly improved the peak EQE value to $9.92 \%$ - the best reported EQE among all colloidal NPL based LEDs (Table S5)..$^{51-53}$ Furthermore, very high brightness values of up to $\sim 46000 \mathrm{~cd} / \mathrm{m}^{2}$ could be obtained from these redemitting colloidal NPL based LEDs with an electroluminescence peak at $650 \mathrm{~nm}$ (Figure 4c). Giovanella et al. reported highly efficient NPL LEDs with an EQE of $8.39 \%$ by using $\mathrm{CdSe} / \mathrm{CdZnS}$ core/shell NPLs, ${ }^{51}$ however, with much lower brightness of $1500 \mathrm{~cd} / \mathrm{m}^{2}$.

Another exciting feature of such colloidal NPL-based LEDs is their low efficiency roll-off at high current densities, enabling very bright electroluminescence. The dependence of EQE on the current density is presented in the Figure $4 \mathrm{~d}$ together with the $J_{90}$ and $J_{50}$ values, which are the current densities corresponding to $10 \%$ and $50 \%$ drops in peak EQE values, respectively. For NPL-LEDs with pure CdS shells, $J_{90}$ and $J_{50}$ values were found to be $\sim 372$ and $1370 \mathrm{~mA} / \mathrm{cm}^{2}$, respectively. Although, in the case of NPL-LEDs with $\mathrm{CdS} / \mathrm{Cd}_{x} \mathrm{Zn}_{1-x} \mathrm{~S}$ 
shells, we observed a similar $J_{90}$ value of $276 \mathrm{~mA} / \mathrm{cm}^{2}$, we obtained a significantly improved $J_{50}$ value of $1729 \mathrm{~mA} / \mathrm{cm}^{2}$, corresponding to the maximum current density reached in the device. The increase of the $J_{50}$ value in NPL-LEDs employing graded-shell-NPLs suggests that the charge balance is improved with the formation of an additional confinement layer. This is also evidenced by the measured EQE of 9.92\%, which is very close to the estimated EQE value of $10.6 \%$. Although our $J_{90}$ and $J_{50}$ values are somewhat lower than the best-performing device based on spherical NCs that is reported in the literature, ${ }^{47}$ our NPL-LEDs outperformed many devices employing colloidal NCs with similar shell compositions in terms of the efficiency roll-off (Figure S8). ${ }^{54-57}$ We also performed a stability test for our NPL LEDs at a constant driving current density, corresponding to the initial luminance $\left(L_{0}\right)$ of $5000 \mathrm{~cd} / \mathrm{m}^{2}$ and measured the device half-lifetime $\left(T_{50}\right.$ $=1.6 \mathrm{~h}$ ), which is defined as the time duration to observe $50 \%$ drop in the luminance (Figure S9). By using the relation of $L_{0}{ }^{n} T_{50}=$ constant and assuming an acceleration factor of $n=$ $1.5,^{58}$ we predicted the half-lifetime of our NPL LED at 100 $\mathrm{cd} / \mathrm{m}^{2}$ as $\sim 560 \mathrm{~h}$. These findings indicate that colloidal NPLs hold great potential for realizing highly stable and bright electroluminescent devices.

\section{CONCLUSIONS}

In conclusion, CdSe NPLs had been overgrown in a one-pot reaction with a $\mathrm{CdS}$ buffer interlayer and a $\mathrm{Cd}_{x} \mathrm{Zn}_{1-x} \mathrm{~S}$ gradient shell, producing highly efficient red-emitting colloidal NPLs (PLQY up to $89 \%$ ) with narrow emission line widths down to $21 \mathrm{~nm}$. These enhanced optical properties enabled us to realize their superior performance in light-emitting applications by demonstrating very low ASE thresholds down to $\sim 40 \mu \mathrm{J} / \mathrm{cm}^{2}$ under nanosecond laser excitation and very bright electroluminescent devices with peak EQEs of $9.92 \%$ - a record among colloidal NPL-based LEDs. Our findings show that, by carefully designing the heterostructures of anisotropically shaped colloidal NPLs, their efficiency can be pushed to the same levels as their isotropically shaped counterparts. In addition to their enhanced optical properties, the anisotropic nature of colloidal NPLs enables the possibility to align their emission dipoles and suppress Auger recombination, making them very promising candidates for the development of nextgeneration optoelectronic devices including high-performance LEDs.

\section{METHODS/EXPERIMENTAL}

Chemicals and Materials. Cadmium nitrate tetrahydrate (Cd$\left(\mathrm{NO}_{3}\right)_{2} \times 4 \mathrm{H}_{2} \mathrm{O}, \geq 99.0 \%$, Fluka), sodium myristate $(98 \%$, TCI), cadmium acetate dihydrate $\left(\mathrm{Cd}(\mathrm{Ac})_{2} \times 2 \mathrm{H}_{2} \mathrm{O}, \geq 99.0 \%\right.$, Fluka), selenium (Se, trace metals basis $99.99 \%, \sim 100$ mesh, Sigma-Aldrich), 1-octanethiol (98\%, ABCR), octadecene (ODE, technical grade $90 \%$, Sigma-Aldrich), oleic acid (OLA, technical grade 90\%, SigmaAldrich), cadmium oxide (CdO, trace metals basis $\geq 99.99 \%$, SigmaAldrich), zinc acetate dihydrate $\left(\mathrm{Zn}(\mathrm{Ac})_{2} \times 2 \mathrm{H}_{2} \mathrm{O}\right)$, oleylamine (OLAm, 80-90\%, approximately C-18 content, Acros Organics), ethanolamine (ACS reagent, $\geq 99.0 \%$, Sigma-Aldrich), tetramethylammonium hydroxide, $25 \% \mathrm{w} / \mathrm{w}$ aqueous solution (TMAH, electronic grade, $99.9999 \%$ metals basis, ABCR). Hexane, methanol, ethanol, ethyl acetate, and dimethyl sulfoxide (DMSO) were purchased from VWR and used without any purification. Anhydrous chlorobenzene (99.8\%) was purchased from Sigma-Aldrich. Patterned indium tin oxide (ITO) substrates were purchased from Kaivo. PEDOT:PSS was purchased from Heraeus (CLEVIOS VP AI 4083). The hole transport material poly(9-vinylcarbazole) (PVK) was purchased from Lumtech. Ag pellets were purchased from Angstrom Engineering.

Synthesis of Nanoplatelets. Preparation of Cadmium Myristate. We prepared cadmium myristate by using the previously published protocol. ${ }^{12}$ For a typical synthesis, $3.13 \mathrm{~g}$ of sodium myristate was dissolved in $250 \mathrm{~mL}$ of methanol, and $1.23 \mathrm{~g}$ of cadmium nitrate tetrahydrate was dissolved in $40 \mathrm{~mL}$ of methanol, separately. After they dissolved and formed clear solutions, the solutions were mixed and stirred for an hour. Afterward, the cadmium myristate was precipitated by centrifugation and dissolved in methanol. The solution was further filtered and washed by using a Buchner funnel to remove unreacted precursors. Finally, the powders were dried under vacuum at $40{ }^{\circ} \mathrm{C}$ overnight and stored outside.

Preparation of Cadmium Oleate. To prepare cadmium oleate, we loaded $1.02 \mathrm{~g}$ of cadmium oxide ( $8 \mathrm{mmol}), 6 \mathrm{~mL}$ of oleic acid, and 34 $\mathrm{mL}$ of ODE into the $100 \mathrm{~mL}$ three-neck flask. After the solution was degassed at $100{ }^{\circ} \mathrm{C}$ for an hour, it was heated to $240{ }^{\circ} \mathrm{C}$ under nitrogen. The reaction mixture was kept at that temperature until the formation of a clear solution. Then, it was cooled to room temperature, and the cadmium oleate $(0.2 \mathrm{M})$ was stored in the nitrogen-filled glovebox.

Preparation of Zinc Oleate. For a typical preparation, we put 1.76 $\mathrm{g}$ of zinc acetate dihydrate $(8 \mathrm{mmol})$ and $6 \mathrm{~mL}$ of oleic acid into the $100 \mathrm{~mL}$ three-neck flask and degassed at $100{ }^{\circ} \mathrm{C}$ for an hour. Then, we added $34 \mathrm{~mL}$ of ODE to the solution and continued degassing for an hour. After the degassing step, we heated the reaction mixture to the $240{ }^{\circ} \mathrm{C}$ under nitrogen and waited until the formation of a clear solution. Finally, it was cooled to room temperature, and the zinc oleate $(0.2 \mathrm{M})$ was stored in the nitrogen-filled glovebox for the shell coating step.

Synthesis of 4 ML Thick CdSe Nanoplatelets. 4 ML thick CdSe NPLs were synthesized according to the previously published protocol. ${ }^{12}$ For a typical synthesis, we placed $340 \mathrm{mg}$ of cadmium myristate, $24 \mathrm{mg}$ of Se, and $30 \mathrm{~mL}$ of ODE into the $100 \mathrm{~mL}$ threeneck flask and degassed at $90{ }^{\circ} \mathrm{C}$ for $30 \mathrm{~min}$. Then, the solution was heated to $240{ }^{\circ} \mathrm{C}$ under nitrogen with a rate of $10-12^{\circ} \mathrm{C} / \mathrm{min}$. When the temperature reached $185-190{ }^{\circ} \mathrm{C}, 80 \mathrm{mg}$ of cadmium acetate dihydrate was swiftly added to the reaction mixture. The reaction solution was kept at $240{ }^{\circ} \mathrm{C}$ for the growth of NPLs. After $10 \mathrm{~min}$ of the growth, the reaction solution was cooled to room temperature with the addition of $1 \mathrm{~mL}$ of oleic acid. For the cleaning step, $5 \mathrm{~mL}$ of hexane was added to the resulting solution, and it was put into a 50 $\mathrm{mL}$ centrifuge tube for centrifugation. After centrifugation, the precipitated part was discarded, and the supernatant part was divided into two parts. With the addition of $6 \mathrm{~mL}$ of ethanol to each part, 4 $\mathrm{mL}$ thick CdSe NPLs were precipitated by centrifugation. Then, the precipitated CdSe NPLs were dissolved in $6 \mathrm{~mL}$ of hexane and cleaned one more time with the addition of $3 \mathrm{~mL}$ of ethanol. Finally, CdSe NPLs were dispersed in hexane and stored outside for the subsequent shell growth.

Synthesis of Core/Shell NPLs. For the growth of CdS shell, we loaded $10 \mathrm{~mL}$ of ODE to the $100 \mathrm{~mL}$ three-neck reaction flask. After it was degassed at $100{ }^{\circ} \mathrm{C}$ for an hour, the solution was heated to 300 ${ }^{\circ} \mathrm{C}$ under nitrogen with a rate of $10-15{ }^{\circ} \mathrm{C} / \mathrm{min}$. When the temperature reached to $150{ }^{\circ} \mathrm{C}$, we began to inject cadmium precursor $(8 \mathrm{~mL}$ of ODE and $8 \mathrm{~mL}$ of cadmium oleate $(0.2 \mathrm{M}))$ and sulfur precursor $(16 \mathrm{~mL}$ of ODE and $336 \mu \mathrm{L}$ of octanethiol) with a rate of $7 \mathrm{~mL} / \mathrm{h}$. After the injection of $0.2 \mathrm{~mL}$ from each precursor, we added the CdSe core NPLs dissolved in $4 \mathrm{~mL}$ of degassed ODE to the reaction mixture. The amount of CdSe NPLs used for the shell coating was equivalent to $4 \mathrm{~mL}$ of CdSe NPL solution with an optical density of 20 at the lowest excitonic peak, assuming a $1 \mathrm{~cm}$ optical path length. This amount of CdSe NPLs was precipitated with the addition of ethanol, transferred to the glovebox, and dissolved in $4 \mathrm{~mL}$ of degassed ODE with the help of sonication. The shell growth proceeded at $300{ }^{\circ} \mathrm{C}$ with the injection of $14 \mathrm{~mL}$ from each precursor. Afterward, the solution was kept at $300{ }^{\circ} \mathrm{C}$ for an additional $20 \mathrm{~min}$ annealing step and cooled to room temperature. For the purification of core/shell NPLs, $5 \mathrm{~mL}$ of hexane was added to the resulting reaction solution, and it was loaded to a $50 \mathrm{~mL}$ centrifuge tube. After 
centrifugation, the precipitated part was kept, and the supernatant was divided into two parts. After the addition of $6 \mathrm{~mL}$ of ethanol to each solution, the NPLs remaining in the supernatant were precipitated. Then, the precipitated NPLs were dissolved in a mixture of $6 \mathrm{~mL}$ of hexane and $0.4 \mathrm{~mL}$ of oleylamine. Subsequently, two more purification steps were performed with ethyl acetate to remove homogeneously nucleated CdS nanocrystals. The resulting NPLs were dissolved in hexane and stored outside for further characterizations and measurements.

For the growth of CdS/Cd $\mathrm{Zn}_{1-x} \mathrm{~S}$, we first started with the growth of the CdS buffer layer by using the same cadmium and sulfur precursors as described above. After the injection of $10 \mathrm{~mL}$ of cadmium and sulfur precursors, the cadmium precursor was replaced with zinc precursor. The zinc precursor was prepared by mixing $3 \mathrm{~mL}$ of zinc oleate, $2 \mathrm{~mL}$ of ODE, and $1 \mathrm{~mL}$ of oleylamine. Then, $4 \mathrm{~mL}$ of zinc and sulfur precursors was injected into the reaction mixture with the same rate of $7 \mathrm{~mL} / \mathrm{h}$ for the growth of $\mathrm{Cd}_{x} \mathrm{Zn}_{1-x} \mathrm{~S}$ gradient shell. After the injection of precursors, the reaction was kept at $300{ }^{\circ} \mathrm{C}$ for an additional $20 \mathrm{~min}$ annealing step, and the reaction was stopped by cooling it to room temperature. The resulting NPLs were washed in a similar way as CdSe/CdS core/shell NPLs. During the optimization, we also studied different injection ratios for the cadmium and zinc precursors by adding the same amount of precursors in total. We found that the core/shell NPLs synthesized with the addition of 10 $\mathrm{mL}$ of cadmium and $4 \mathrm{~mL}$ of zinc precursors exhibit the higher photoluminescence quantum yield (PLQY).

For the synthesis of $\mathrm{CdSe} / \mathrm{ZnS}$ core/shell NPLs, we used the zinc and sulfur precursors, and they were injected with the same injection rate. The zinc precursor was prepared by mixing $8 \mathrm{~mL}$ of zinc oleate, 7 $\mathrm{mL}$ of ODE, and $1 \mathrm{~mL}$ of oleylamine. The shell growth temperature and growth time were the same as the previous synthesis.

Optical and Structural Characterization of Colloidal NPLs. Absorption spectra of colloidal NPLs were collected with a Jasco V-670 UV-vis-NIR spectrometer (NIR = near-infrared) operating in the transmission mode. Photoluminescence spectra of colloidal NPLs were recorded with a Fluorolog iHR 320 Horiba Jobin Yvon spectrofluorimeter equipped with photomultiplier tube (PMT) detector. Photoluminescence quantum yield of the solutions and films was measured by using a Hamamatsu Quantaurus QY spectrometer (C11347-11) equipped with an integrating sphere. Time-resolved photoluminescence measurements were performed by using FluoTime 300 spectrometer from PicoQuant Gmbh. X-ray diffraction patterns were recorded by using an STOE IPDS II single-crystal diffractometer equipped with image plate detector, a sealed tube with $\mathrm{Cu} \mathrm{K} \alpha$ radiation and graphite monochromator, and converted to the "classical" powder diffraction pattern by using the X-AREA software. Transmission electron microscopy images were acquired by using a Hitachi HT7700 microscope operating at $100 \mathrm{kV}$. Scanning transmission electron microscopy and energy-dispersive X-ray spectroscopy were performed by using FEI Talos F200X microscope operating at $200 \mathrm{kV}$.

Amplified Spontaneous Emission (ASE) Measurements. For studying the optical gain characteristics of NPLs, we prepared their solid films by spin coating on bare quartz substrates. ASE measurements were performed by using femtosecond and nanosecond excitation lasers. To minimize the measurement error of tightly focused laser spot size on the sample during the lasing experiments, the samples were always situated before the focal point of the lens, and a slightly defocused laser beam was used. Beam profile on the sample was measured from the sample PL under weak excitation (below the ASE threshold) with a microscope to calculate the pulse energy density starting from the pulse energy.

For nanosecond excitation, ASE experiments were performed by using an LTB MNL 100 nitrogen pump laser delivering $3 \mathrm{~ns}$ pulses at $337 \mathrm{~nm}$, with a peak energy up to $155 \mu \mathrm{J}$, with a repetition rate of 10 $\mathrm{Hz}$. The pump laser was focused onto a stripe by a cylindrical lens having a focal length of $150 \mathrm{~mm}$. A metal slit with a $4 \mathrm{~mm}$ opening was used to define the stripe length, and it was placed after the lens. For the accurate measurement of the width of the stripe, the sample was placed a few millimeters before the lens focus. The dimensions of the rectangular stripe were measured as $4 \mathrm{~mm}$ in length and $80 \mu \mathrm{m}$ in width. The pump laser energy was adjusted with a continuously variable neutral filter and measured by an energy meter triggered by the laser with a sensitivity allowing us to measure down to $0.28 \mu \mathrm{J}$ per pulse. In this experiment we used a minimum energy of $5 \mu \mathrm{J}$, to have sensitivity errors always below 5.5\%. Lower energies are obtained with two further neutral filters, positioned after the energy meter, with calibrated transmittance, allowing to reach a minimum energy of 18 $\mathrm{nJ}$, corresponding to $4 \mu \mathrm{J} / \mathrm{cm}^{2}$ with a relative error of $\sim 5.5 \%$. The relative error on the ASE thresholds is of the order of $0.5 \%$.The sample emission was collected from the edge of samples after waveguiding along the pumped stripe, spectrally dispersed by an Acton 750 spectrometer and detected by an Andocr Peltier cooled charge-coupled device (CCD). The spectral resolution was $0.5 \mathrm{~nm}$.

For femtosecond excitation, ASE measurements were done by using a laser system consisting of an oscillator (Vitesse 800) and an amplifier (Legend Elite), both from Coherent Inc., with a frequencydoubling $\beta$-barium borate (BBO) crystal; it yielded $100 \mathrm{fs}$ pulses at $400 \mathrm{~nm}$, with a repetition rate of $1 \mathrm{kHz}$ and pulse energy of up to $4 \mu \mathrm{J}$. The pump laser was focused onto a stripe by a plano-convex cylindrical lens with a focal length of $75 \mathrm{~mm}$. A metal slit with a $2 \mathrm{~mm}$ opening was placed after the lens to determine the stripe length. To accurately measure the width of the stripe, the sample was situated a few millimeters before the lens focus. The dimensions of the stripe were determined as $2 \mathrm{~mm}$ in length and $50 \mu \mathrm{m}$ in width. The sample emission was collected from the edges by a fiber-coupled spectrometer CCS200 CCD (Thorlabs).

Fabrication and Characterization of LEDs. Synthesis of $\mathrm{ZnO}$ Nanocrystals (NCs). ZnO NCs were synthesized by using the published protocol with slight modifications. ${ }^{44}$ For a typical synthesis, $657 \mathrm{mg}$ of zinc acetate dihydrate $(3 \mathrm{mmol})$ was dissolved in $30 \mathrm{~mL}$ of DMSO at room temperature. Afterward, a mixture of ethanol (8.5 $\mathrm{mL})$ and $25 \mathrm{wt} \%$ TMAH solution in water $(1.5 \mathrm{~mL})$ was injected with a rate of $2.0 \mathrm{~mL} / \mathrm{min}$. The solution was stirred at ambient conditions for an hour. Then, $\mathrm{ZnO}$ NCs were precipitated with the addition of ethyl acetate and dispersed in ethanol. For improving the colloidal stability of the $\mathrm{ZnO} N C s, 200 \mu \mathrm{L}$ of ethanolamine was added into the solution of $\mathrm{ZnO}$ and stirred for an hour. Finally, the resulting $\mathrm{ZnO} \mathrm{NCs}$ were washed with ethyl acetate twice and dissolved in ethanol.

Fabrication of LEDs. For the fabrication of NPL-LEDs, we used patterned ITO substrates. ITO substrates were cleaned by sonication in various solvents in the following order; a mixture of deionized water and detergent solution, acetone, and isopropyl alcohol for 20 min. Subsequently, UV ozone treatment was used for the substrates for $10 \mathrm{~min}$. Then, PEDOT:PSS solution was spin-coated on ITOcovered substrates at $4000 \mathrm{rpm}$ for $30 \mathrm{~s}$, and the substrates were annealed at $150{ }^{\circ} \mathrm{C}$ for $30 \mathrm{~min}$ under ambient conditions. After the annealing step, the substrates were transferred to the nitrogen-filled glovebox for the deposition of subsequent layers. For the hole transport layer, we used PVK dissolved in anhydrous chlorobenzene with a concentration of $5 \mathrm{mg} / \mathrm{mL}$. It was spin-coated at $2000 \mathrm{rpm}$ for $1 \mathrm{~min}$ and annealed at $150{ }^{\circ} \mathrm{C}$ for $30 \mathrm{~min}$. After that, core/shell NPLs dissolved in hexane $(\sim 10 \mathrm{mg} / \mathrm{mL})$ were spin-coated at $2000 \mathrm{rpm}$ for $1 \mathrm{~min}$. It was followed by the deposition of $\mathrm{ZnO}$ nanoparticles dissolved in ethanol $(30 \mathrm{mg} / \mathrm{mL})$ at $2000 \mathrm{rpm}$ for $1 \mathrm{~min}$. After the coating of NPL and $\mathrm{ZnO}$ layers, no annealing treatment was applied. Afterward, the substrates were placed into a vacuum chamber at $1 \times$ $10^{-7}$ mbar for the thermal evaporation of $100 \mathrm{~nm}$ thick $\mathrm{Ag}$ cathode layer through a shadow mask. After the deposition of the metal contact layer, the devices were encapsulated inside the glovebox by using UV-curable epoxy. The measurements were performed outside the glovebox. An active pixel area of devices was determined as 16 $\mathrm{mm}^{2}$ from the overlap of ITO and Ag.

Characterization of LEDs. We measured the external quantum efficiencies of the fabricated devices by using a method suggested by Forrest et al. ${ }^{59}$ A calibrated Si photodiode $(1 \times 1 \mathrm{~cm}$, Thorlabs FDS$1010 \mathrm{CAL}$ ) was placed on top of the active pixel to collect the emitted light from the active pixel, which is considerably larger than the size of our pixel $(4 \times 4 \mathrm{~mm})$. The edges of the substrate are far away from 
the active pixel, and thus we collected the emission only escaping from the active pixel in the forward direction. The current densityvoltage-luminescence characteristics of the fabricated devices were measured by using a Keysight B2902a source measurement unit and the calibrated $\mathrm{Si}$ photodiode. Separately, the electroluminescence (EL) spectra of the LEDs were collected by using a fiber-coupled spectrometer (CCS200 CCD spectrometer, Thorlabs) and were used to take into account sensitivity of the photodiode.

\section{ASSOCIATED CONTENT}

\section{S Supporting Information}

The Supporting Information is available free of charge at https://pubs.acs.org/doi/10.1021/acsnano.9b05313.

The optical and structural characterization of NPLs; the optical gain experiments with NPLs; ASE stability measurements; PLQY measurements; time-resolved photoluminescence measurements and analysis; literature survey of colloidal NPL and colloidal NC based LEDs; operational stability of NPL LEDs (PDF)

\section{AUTHOR INFORMATION}

\section{Corresponding Author}

*E-mail: mvkovalenko@ethz.ch.

\section{ORCID}

Yevhen Shynkarenko: 0000-0002-1587-1752

Marco Anni: 0000-0002-1651-0166

Sergii Yakunin: 0000-0002-6409-0565

Maria Luisa De Giorgi: 0000-0002-3774-6477

Maksym V. Kovalenko: 0000-0002-6396-8938

\section{Notes}

The authors declare no competing financial interest.

\section{ACKNOWLEDGMENTS}

M.K and Y.K. acknowledge financial support from the Swiss Federal Commission for Technology and Innovation (CTINo. 25493.3) and the European Union's Horizon 2020 research and innovation program under the Marie Skłodowska-Curie Grant No. 798697. We acknowledge the support of the Scientific Center for Optical and Electron Microscopy (ScopeM) of the Swiss Federal Institute of Technology ETHZ. We thank Prof. M. Fiebig and his research group for access to their femtosecond laser and for experimental assistance and B. M. Benin for reading the manuscript.

\section{REFERENCES}

(1) Lhuillier, E.; Pedetti, S.; Ithurria, S.; Nadal, B.; Heuclin, H.; Dubertret, B. Two-Dimensional Colloidal Metal Chalcogenides Semiconductors: Synthesis, Spectroscopy, and Applications. Acc. Chem. Res. 2015, 48, 22-30.

(2) Ithurria, S.; Dubertret, B. Quasi 2D Colloidal CdSe Platelets with Thicknesses Controlled at the Atomic Level. J. Am. Chem. Soc. 2008, 130, 16504-16505.

(3) Ithurria, S.; Tessier, M. D.; Mahler, B.; Lobo, R. P. S. M.; Dubertret, B.; Efros, A. L. Colloidal Nanoplatelets with TwoDimensional Electronic Structure. Nat. Mater. 2011, 10, 936-941.

(4) Yeltik, A.; Delikanli, S.; Olutas, M.; Kelestemur, Y.; Guzelturk, B.; Demir, H. V. Experimental Determination of the Absorption Cross-Section and Molar Extinction Coefficient of Colloidal CdSe Nanoplatelets. J. Phys. Chem. C 2015, 119, 26768-26775.

(5) Tessier, M. D.; Javaux, C.; Maksimovic, I.; Loriette, V.; Dubertret, B. Spectroscopy of Single CdSe Nanoplatelets. ACS Nano 2012, 6, 6751-6758.
(6) Kunneman, L. T.; Tessier, M. D.; Heuclin, H.; Dubertret, B.; Aulin, Y. V.; Grozema, F. C.; Schins, J. M.; Siebbeles, L. D. A. Bimolecular Auger Recombination of Electron-Hole Pairs in TwoDimensional CdSe and CdSe/CdZnS Core/Shell Nanoplatelets. J. Phys. Chem. Lett. 2013, 4, 3574-3578.

(7) She, C.; Fedin, I.; Dolzhnikov, D. S.; Demortière, A.; Schaller, R. D.; Pelton, M.; Talapin, D. V. Low-Threshold Stimulated Emission Using Colloidal Quantum Wells. Nano Lett. 2014, 14, 2772-2777.

(8) Guzelturk, B.; Kelestemur, Y.; Olutas, M.; Delikanli, S.; Demir, H. V. Amplified Spontaneous Emission and Lasing in Colloidal Nanoplatelets. ACS Nano 2014, 8, 6599-6605.

(9) Guzelturk, B.; Pelton, M.; Olutas, M.; Demir, H. V. Giant Modal Gain Coefficients in Colloidal II-VI Nanoplatelets. Nano Lett. 2019, 19, 277-282.

(10) Chen, Z.; Nadal, B.; Mahler, B.; Aubin, H.; Dubertret, B. Quasi2D Colloidal Semiconductor Nanoplatelets for Narrow Electroluminescence. Adv. Funct. Mater. 2014, 24, 295-302.

(11) Zhang, F.; Wang, S.; Wang, L.; Lin, Q.; Shen, H.; Cao, W.; Yang, C.; Wang, H.; Yu, L.; Du, Z.; Xue, J.; Li, L. S. Super Color Purity Green Quantum Dot Light-Emitting Diodes Fabricated by Using CdSe/CdS Nanoplatelets. Nanoscale 2016, 8, 12182-12188.

(12) Tessier, M. D.; Spinicelli, P.; Dupont, D.; Patriarche, G.; Ithurria, S.; Dubertret, B. Efficient Exciton Concentrators Built from Colloidal Core/Crown CdSe/CdS Semiconductor Nanoplatelets. Nano Lett. 2014, 14, 207-213.

(13) Mahler, B.; Nadal, B.; Bouet, C.; Patriarche, G.; Dubertret, B. Core/Shell Colloidal Semiconductor Nanoplatelets. J. Am. Chem. Soc. 2012, 134, 18591-18598.

(14) Kelestemur, Y.; Olutas, M.; Delikanli, S.; Guzelturk, B.; Akgul, M. Z.; Demir, H. V. Type-II Colloidal Quantum Wells: CdSe/CdTe Core/Crown Heteronanoplatelets. J. Phys. Chem. C 2015, 119, 21772185.

(15) Kelestemur, Y.; Guzelturk, B.; Erdem, O.; Olutas, M.; Erdem, T.; Usanmaz, C. F.; Gungor, K.; Demir, H. V. CdSe/CdSe 1- x Te x Core/Crown Heteronanoplatelets: Tuning the Excitonic Properties without Changing the Thickness. J. Phys. Chem. C 2017, 121, 46504658.

(16) Delikanli, S.; Guzelturk, B.; Hernández-Martínez, P. L.; Erdem, T.; Kelestemur, Y.; Olutas, M.; Akgul, M. Z.; Demir, H. V. Continuously Tunable Emission in Inverted Type-I CdS/CdSe Core/Crown Semiconductor Nanoplatelets. Adv. Funct. Mater. 2015, 25, 4282-4289.

(17) Ithurria, S.; Talapin, D. V. Colloidal Atomic Layer Deposition (c-ALD) Using Self-Limiting Reactions at Nanocrystal Surface Coupled to Phase Transfer between Polar and Nonpolar Media. J. Am. Chem. Soc. 2012, 134, 18585-18590.

(18) Rowland, C. E.; Fedin, I.; Diroll, B. T.; Liu, Y.; Talapin, D. V.; Schaller, R. D. Elevated Temperature Photophysical Properties and Morphological Stability of CdSe and CdSe/CdS Nanoplatelets. J. Phys. Chem. Lett. 2018, 9, 286-293.

(19) Yadav, S.; Singh, A.; Thulasidharan, L.; Sapra, S. Surface Decides the Photoluminescence of Colloidal CdSe Nanoplatelets Based Core/Shell Heterostructures. J. Phys. Chem. C 2018, 122, 820829.

(20) Kelestemur, Y.; Guzelturk, B.; Erdem, O.; Olutas, M.; Gungor, K.; Demir, H. V. Platelet-in-Box Colloidal Quantum Wells: CdSe/ CdS@CdS Core/Crown@Shell Heteronanoplatelets. Adv. Funct. Mater. 2016, 26, 3570-3579.

(21) Shendre, S.; Delikanli, S.; Li, M.; Dede, D.; Pan, Z.; Ha, S. T.; Fu, Y. H.; Hernández-Martínez, P. L.; Yu, J.; Erdem, O.; Kuznetsov, A. I.; Dang, C.; Sum, T. C.; Demir, H. V. Ultrahigh-Efficiency Aqueous Flat Nanocrystals of CdSe/CdS@Cd 1-x Zn x S Colloidal Core/Crown@alloyed-Shell Quantum Wells. Nanoscale 2019, 11, $301-310$.

(22) Rossinelli, A. A.; Riedinger, A.; Marqués-Gallego, P.; Knüsel, P. N.; Antolinez, F. V.; Norris, D. J. High-Temperature Growth of Thick-Shell CdSe/CdS Core/Shell Nanoplatelets. Chem. Commun. 2017, 53, 9938-9941. 
(23) Altintas, Y.; Quliyeva, U.; Gungor, K.; Erdem, O.; Kelestemur, Y.; Mutlugun, E.; Kovalenko, M. V.; Demir, H. V. Highly Stable, Near-Unity Efficiency Atomically Flat Semiconductor Nanocrystals of $\mathrm{CdSe} / \mathrm{ZnS}$ Hetero-Nanoplatelets Enabled by ZnS-Shell Hot-Injection Growth. Small 2019, 15, 1-11.

(24) Fedin, I.; Talapin, D. V. Colloidal CdSe Quantum Rings. J. Am. Chem. Soc. 2016, 138, 9771-9774.

(25) Polovitsyn, A.; Dang, Z.; Movilla, J. L.; Martín-García, B.; Khan, A. H.; Bertrand, G. H. V.; Brescia, R.; Moreels, I. Synthesis of AirStable CdSe/ZnS Core-Shell Nanoplatelets with Tunable Emission Wavelength. Chem. Mater. 2017, 29, 5671-5680.

(26) Rossinelli, A. A.; Rojo, H.; Mule, A. S.; Aellen, M.; Cocina, A.; De Leo, E.; Schäublin, R.; Norris, D. J. Compositional Grading for Efficient and Narrowband Emission in CdSe-Based Core/Shell Nanoplatelets. Chem. Mater. 2019, 31, 9567.

(27) Altintas, Y.; Gungor, K.; Gao, Y.; Sak, M.; Quliyeva, U.; Bappi, G.; Mutlugun, E.; Sargent, E. H.; Demir, H. V. Giant Alloyed Hot Injection Shells Enable Ultralow Optical Gain Threshold in Colloidal Quantum Wells. ACS Nano 2019, 13, 10662-10670.

(28) Singh, S.; Tomar, R.; ten Brinck, S.; De Roo, J.; Geiregat, P.; Martins, J. C.; Infante, I.; Hens, Z. Colloidal CdSe Nanoplatelets, A Model for Surface Chemistry/Optoelectronic Property Relations in Semiconductor Nanocrystals. J. Am. Chem. Soc. 2018, 140, 1329213300 .

(29) Tessier, M. D.; Mahler, B.; Nadal, B.; Heuclin, H.; Pedetti, S.; Dubertret, B. Spectroscopy of Colloidal Semiconductor Core/Shell Nanoplatelets with High Quantum Yield. Nano Lett. 2013, 13, 33213328.

(30) Chen, O.; Zhao, J.; Chauhan, V. P.; Cui, J.; Wong, C.; Harris, D. K.; Wei, H.; Han, H.-S.; Fukumura, D.; Jain, R. K.; Bawendi, M. G. Compact High-Quality CdSe-CdS Core-Shell Nanocrystals with Narrow Emission Linewidths and Suppressed Blinking. Nat. Mater. 2013, 12, 445-451.

(31) Greytak, A. B.; Allen, P. M.; Liu, W.; Zhao, J.; Young, E. R.; Popović, Z.; Walker, B. J.; Nocera, D. G.; Bawendi, M. G. Alternating Layer Addition Approach to CdSe/CdS Core/Shell Quantum Dots with Near-Unity Quantum Yield and High On-Time Fractions. Chem. Sci. 2012, 3, 2028-2034.

(32) Boldt, K.; Kirkwood, N.; Beane, G. A.; Mulvaney, P. Synthesis of Highly Luminescent and Photo-Stable, Graded Shell CdSe/Cd x Zn 1- x S Nanoparticles by In Situ Alloying. Chem. Mater. 2013, 25, 4731-4738.

(33) Bae, W. K.; Char, K.; Hur, H.; Lee, S. Single-Step Synthesis of Quantum Dots with Chemical Composition Gradients. Chem. Mater. 2008, 20, 531-539.

(34) Pelton, M. Carrier Dynamics, Optical Gain, and Lasing with Colloidal Quantum Wells. J. Phys. Chem. C 2018, 122, 10659-10674.

(35) She, C.; Fedin, I.; Dolzhnikov, D. S.; Dahlberg, P. D.; Engel, G. S.; Schaller, R. D.; Talapin, D. V. Red, Yellow, Green, and Blue Amplified Spontaneous Emission and Lasing Using Colloidal CdSe Nanoplatelets. ACS Nano 2015, 9, 9475-9485.

(36) Guzelturk, B.; Kelestemur, Y.; Olutas, M.; Li, Q.; Lian, T.; Demir, H. V. High-Efficiency Optical Gain in Type-II Semiconductor Nanocrystals of Alloyed Colloidal Quantum Wells. J. Phys. Chem. Lett. 2017, 8, 5317-5324.

(37) Kelestemur, Y.; Dede, D.; Gungor, K.; Usanmaz, C. F.; Erdem, O.; Demir, H. V. Alloyed Heterostructures of CdSexS1-x Nanoplatelets with Highly Tunable Optical Gain Performance. Chem. Mater. 2017, 29, 4857-4865.

(38) Grim, J. Q.; Christodoulou, S.; Di Stasio, F.; Krahne, R.; Cingolani, R.; Manna, L.; Moreels, I. Continuous-Wave Biexciton Lasing at Room Temperature Using Solution-Processed Quantum Wells. Nat. Nanotechnol. 2014, 9, 891-895.

(39) Kelestemur, Y.; Cihan, A. F.; Guzelturk, B.; Demir, H. V. TypeTunable Amplified Spontaneous Emission from Core-Seeded CdSe/ CdS Nanorods Controlled by Exciton-Exciton Interaction. Nanoscale 2014, 6, 8509-8514.

(40) Cihan, A. F.; Kelestemur, Y.; Guzelturk, B.; Yerli, O.; Kurum, U.; Yaglioglu, H. G.; Elmali, A.; Demir, H. V. Attractive versus
Repulsive Excitonic Interactions of Colloidal Quantum Dots Control Blue- to Red-Shifting (and Non-Shifting) Amplified Spontaneous Emission. J. Phys. Chem. Lett. 2013, 4, 4146-4152.

(41) Colvin, V. L.; Schlamp, M. C.; Alivisatos, A. P. Light-Emitting Diodes Made from Cadmium Selenide Nanocrystals and a Semiconducting Polymer. Nature 1994, 370, 354-357.

(42) Shirasaki, Y.; Supran, G. J.; Bawendi, M. G.; Bulović, V. Emergence of Colloidal Quantum-Dot Light-Emitting Technologies. Nat. Photonics 2013, 7, 13-23.

(43) Pietryga, J. M.; Park, Y.-S.; Lim, J.; Fidler, A. F.; Bae, W. K.; Brovelli, S.; Klimov, V. I. Spectroscopic and Device Aspects of Nanocrystal Quantum Dots. Chem. Rev. 2016, 116, 10513-10622.

(44) Dai, X.; Zhang, Z.; Jin, Y.; Niu, Y.; Cao, H.; Liang, X.; Chen, L.; Wang, J.; Peng, X. Solution-Processed, High-Performance LightEmitting Diodes Based on Quantum Dots. Nature 2014, 515, 96-99.

(45) Cheng, T.; Wang, F.; Sun, W.; Wang, Z.; Zhang, J.; You, B.; Li, Y.; Hayat, T.; Alsaed, A.; Tan, Z. High-Performance Blue Quantum Dot Light-Emitting Diodes with Balanced Charge Injection. $A d v$. Electron. Mater. 2019, 5, 1-9.

(46) Li, X.; Zhao, Y.-B.; Fan, F.; Levina, L.; Liu, M.; QuinteroBermudez, R.; Gong, X.; Quan, L. N.; Fan, J.; Yang, Z.; Hoogland, S.; Voznyy, O.; Lu, Z.-H.; Sargent, E. H. Bright Colloidal Quantum Dot Light-Emitting Diodes Enabled by Efficient Chlorination. Nat. Photonics 2018, 12, 159-164.

(47) Lim, J.; Park, Y.-S.; Wu, K.; Yun, H. J.; Klimov, V. I. DroopFree Colloidal Quantum Dot Light-Emitting Diodes. Nano Lett. 2018, 18, 6645-6653.

(48) Bozyigit, D.; Wood, V. Challenges and Solutions for HighEfficiency Quantum Dot-Based LEDs. MRS Bull. 2013, 38, 731-736.

(49) Gao, Y.; Weidman, M. C.; Tisdale, W. A. CdSe Nanoplatelet Films with Controlled Orientation of Their Transition Dipole Moment. Nano Lett. 2017, 17, 3837-3843.

(50) Kim, W. D.; Kim, D.; Yoon, D.-E.; Lee, H.; Lim, J.; Bae, W. K.; Lee, D. C. Pushing the Efficiency Envelope for Semiconductor Nanocrystal-Based Electroluminescence Devices Using Anisotropic Nanocrystals. Chem. Mater. 2019, 31, 3066-3082.

(51) Giovanella, U.; Pasini, M.; Lorenzon, M.; Galeotti, F.; Lucchi, C.; Meinardi, F.; Luzzati, S.; Dubertret, B.; Brovelli, S. Efficient Solution-Processed Nanoplatelet-Based Light-Emitting Diodes with High Operational Stability in Air. Nano Lett. 2018, 18, 3441-3448.

(52) Liu, B.; Delikanli, S.; Gao, Y.; Dede, D.; Gungor, K.; Demir, H. V. Nanocrystal Light-Emitting Diodes Based on Type II Nanoplatelets. Nano Energy 2018, 47, 115-122.

(53) Zhang, F.; Wang, S.; Wang, L.; Lin, Q.; Shen, H.; Cao, W.; Yang, C.; Wang, H.; Yu, L.; Du, Z.; Xue, J.; Li, L. S. Super Color Purity Green Quantum Dot Light-Emitting Diodes Fabricated by Using CdSe/CdS Nanoplatelets. Nanoscale 2016, 8, 12182-12188.

(54) Bae, W. K.; Park, Y.-S.; Lim, J.; Lee, D.; Padilha, L. A.; McDaniel, H.; Robel, I.; Lee, C.; Pietryga, J. M.; Klimov, V. I. Controlling the Influence of Auger Recombination on the Performance of Quantum-Dot Light-Emitting Diodes. Nat. Commun. 2013, 4, 2661.

(55) Lim, J.; Jeong, B. G.; Park, M.; Kim, J. K.; Pietryga, J. M.; Park, Y.-S.; Klimov, V. I.; Lee, C.; Lee, D. C.; Bae, W. K. Influence of Shell Thickness on the Performance of Light-Emitting Devices Based on CdSe/Zn 1-X Cd X S Core/Shell Heterostructured Quantum Dots. Adv. Mater. 2014, 26, 8034-8040.

(56) Nam, S.; Oh, N.; Zhai, Y.; Shim, M. High Efficiency and Optical Anisotropy in Double-Heterojunction Nanorod LightEmitting Diodes. ACS Nano 2015, 9, 878-885.

(57) Mashford, B. S.; Stevenson, M.; Popovic, Z.; Hamilton, C.; Zhou, Z.; Breen, C.; Steckel, J.; Bulovic, V.; Bawendi, M.; CoeSullivan, S.; Kazlas, P. T. High-Efficiency Quantum-Dot LightEmitting Devices with Enhanced Charge Injection. Nat. Photonics 2013, 7, 407-412.

(58) Wellmann, P.; Hofmann, M.; Zeika, O.; Werner, A.; Birnstock, J.; Meerheim, R.; He, G.; Walzer, K.; Pfeiffer, M.; Leo, K. HighEfficiency $p-i-n$ Organic Light-Emitting Diodes with Long Lifetime. J. Soc. Inf. Disp. 2005, 13, 393. 
(59) Forrest, S. R.; Bradley, D. D. C.; Thompson, M. E. Measuring the Efficiency of Organic Light-Emitting Devices. Adv. Mater. 2003, $15,1043-1048$. 